ARTICLE

\title{
Terahertz-driven linear electron acceleration
}

\author{
Emilio A. Nanni ${ }^{1}$, Wenqian R. Huang ${ }^{1}$, Kyung-Han Hong ${ }^{1}$, Koustuban Ravi ${ }^{1}$, Arya Fallahi ${ }^{2,3}$, Gustavo Moriena ${ }^{4}$, \\ R.J. Dwayne Miller ${ }^{3,4,5}$ \& Franz X. Kärtner $1,2,3,6$
}

The cost, size and availability of electron accelerators are dominated by the achievable accelerating gradient. Conventional high-brightness radio-frequency accelerating structures operate with $30-50 \mathrm{MeVm}^{-1}$ gradients. Electron accelerators driven with optical or infrared sources have demonstrated accelerating gradients orders of magnitude above that achievable with conventional radio-frequency structures. However, laser-driven wakefield accelerators require intense femtosecond sources and direct laser-driven accelerators suffer from low bunch charge, sub-micron tolerances and sub-femtosecond timing requirements due to the short wavelength of operation. Here we demonstrate linear acceleration of electrons with keV energy gain using optically generated terahertz pulses. Terahertz-driven accelerating structures enable high-gradient electron/proton accelerators with simple accelerating structures, high repetition rates and significant charge per bunch. These ultra-compact terahertz accelerators with extremely short electron bunches hold great potential to have a transformative impact for free electron lasers, linear colliders, ultrafast electron diffraction, $\mathrm{X}$-ray science and medical therapy with $\mathrm{X}$-rays and electron beams.

\footnotetext{
${ }^{1}$ Department of Electrical Engineering and Computer Science, Research Laboratory of Electronics, Massachusetts Institute of Technology, Cambridge, Massachusetts 02139, USA. ${ }^{2}$ Center for Free-Electron Laser Science and The Hamburg Center for Ultrafast Imaging, Hamburg 22607, Germany. ${ }^{3}$ Deutsches Elektronen Synchrotron, Ultrafast Optics and X-rays Division, Hamburg 22607, Germany. ${ }^{4}$ Department of Chemistry and Physics, University of Toronto, Toronto, Ontario M5S, Canada. ${ }^{5}$ Max Planck Institute for the Structure and Dynamics of Matter, Hamburg 22607, Germany. ${ }^{6}$ Department of Physics, University of Hamburg, Hamburg 20148, Germany. Correspondence and requests for materials should be addressed to E.A.N. (email: enanni@alum.mit.edu) or to F.X.K. (email: franz.kaertner@cfel.de).
} 
$t$ radio-frequency $(\mathrm{RF})$ frequencies where conventional sources (klystrons and so on) are efficient, surface electric field gradients in accelerating structures are limited by RF-induced plasma breakdown ${ }^{1,2}$. Empirically, the breakdown threshold has been found $d^{3,4}$ to scale as $E_{\mathrm{s}} \propto f^{1 / 2} \tau^{-1 / 4}$, where $E_{\mathrm{s}}$ is the surface electric field, $f$ is the frequency of operation and $\tau$ is the pulse length indicating that higher frequencies and shorter pulse durations are clearly beneficial. This empirical scaling is limited by the onset of field emission due to the electric field on material surfaces. For most common accelerating materials, this onset is in the $10 \mathrm{~s}$ of $\mathrm{GV} \mathrm{m}^{-1}$ range $^{5,6}$. In addition, low frequencies (that is, $\mathrm{GHz}$ ) inherently require long $\mathrm{RF}$ pulses because a single RF cycle is long (on the order of ns) and traditional sources work most efficiently when operating over a very narrow frequency spectrum (that is, long pulse length). Practically, this results in a significant amount of average power coupled into the structure if a high repetition rate is used.

If high accelerating gradients are desired, presently available ultrafast near infrared (NIR) terawatt and petawatt laser technology based on chirped pulse amplification allows for multi-GeV m ${ }^{-1}$ gradients $^{7-16}$. However, due to the short wavelength, infrared optical pulses prove to be difficult to use for the direct acceleration of electrons with significant charge per bunch, which is an important parameter. For example, the coherent emission in a free electron laser (FEL) from an electron bunch scales with the square of the number of electrons in the bunch $^{1}$. To prevent emittance growth and increased energy spread, the electron bunch needs to occupy a small fraction of the optical cycle. Even for a long-infrared wavelength of $10 \mu \mathrm{m}, 1^{\circ}$ of phase in the optical wavelength corresponds to only $\sim 28 \mathrm{~nm}$. Another practical concern would be the timing precision between the optical cycle and the arrival of the electron bunch. For example, $1^{\circ}$ phase jitter, commonly required for operational accelerators, requires $<100$ as timing jitter between the optical pulse and the electron bunch, which is challenging to maintain over extended distances. Difficulties increase further when considering the available options for guiding the optical light to decrease the phase velocity to match the electron beam. A guided mode at a wavelength of $10 \mu \mathrm{m}$ would require sub-micron precision for aligning the electron bunch and the optical waveguide.

Alternatively, laser plasma wakefield accelerators have demonstrated $\mathrm{GeV} \mathrm{m}^{-1}$ accelerating gradients ${ }^{7,9,17}$ with 100 terawatt to petawatt (TW-PW) low repetition rate sources with percent-level energy spread and jitter for the electron bunch. The complexity of these plasma wakefield accelerators is significant because the acceleration mechanism relies on plasma bubbles, which are easily subject to instabilities. Also, power scaling of the required high-energy femtosecond pulse sources is challenging.

$\mathrm{THz}$ frequencies provide the best of both worlds ${ }^{18-21}$. On one hand, the wavelength is long enough that we can fabricate waveguides with conventional machining techniques, provide accurate timing and accommodate a significant amount of charge per bunch. At $0.3 \mathrm{THz}$, the wavelength is $1 \mathrm{~mm}$ and $1^{\circ}$ of phase a

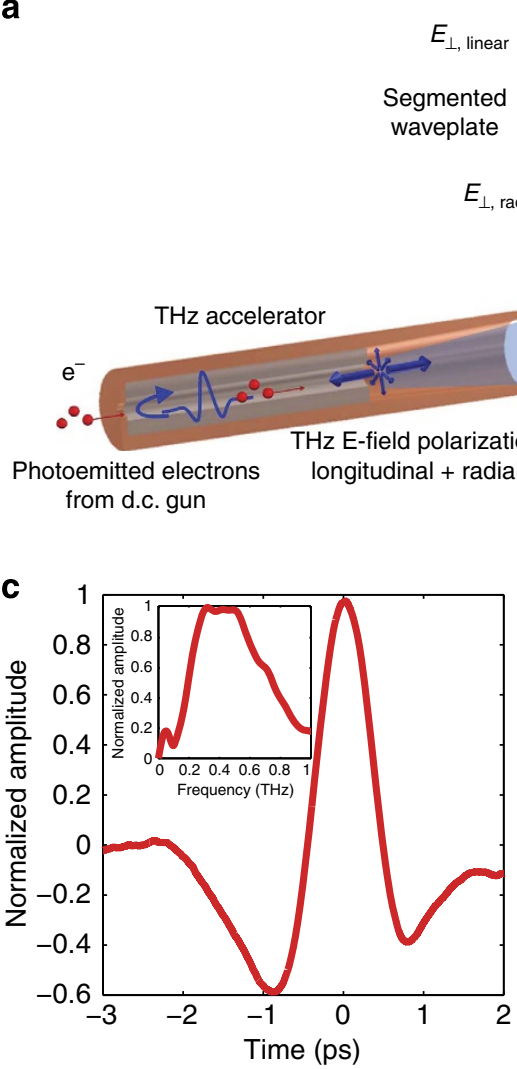

$E_{\perp, \text { linear }}$

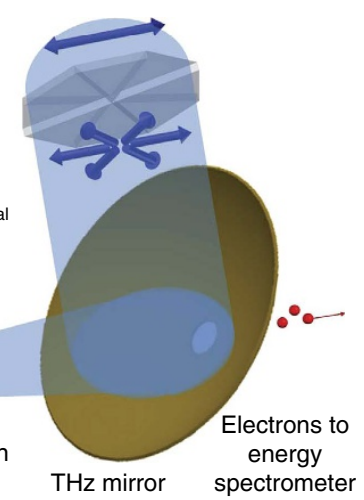

b

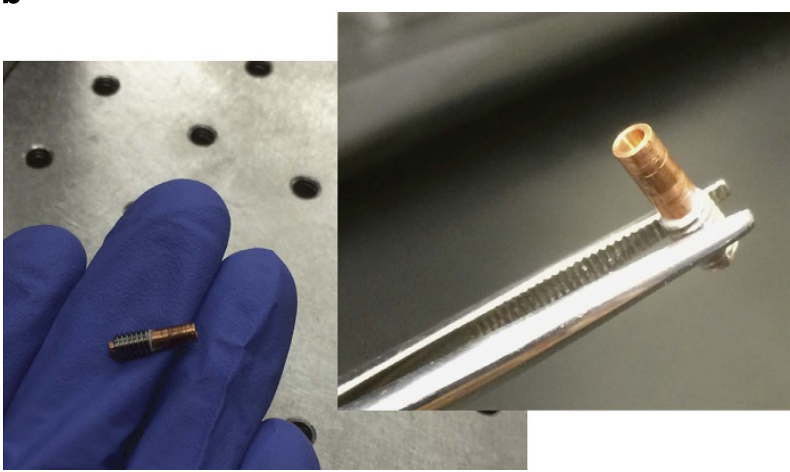

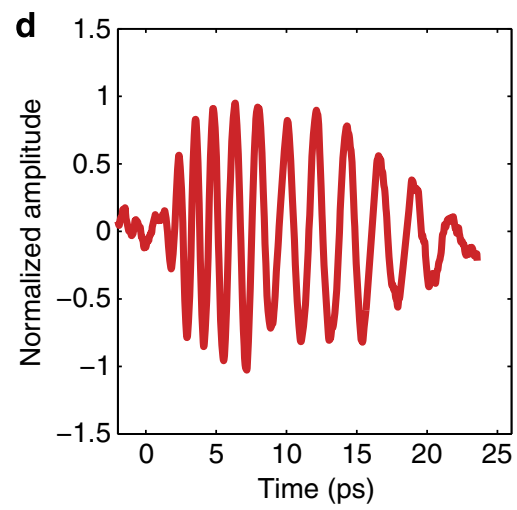

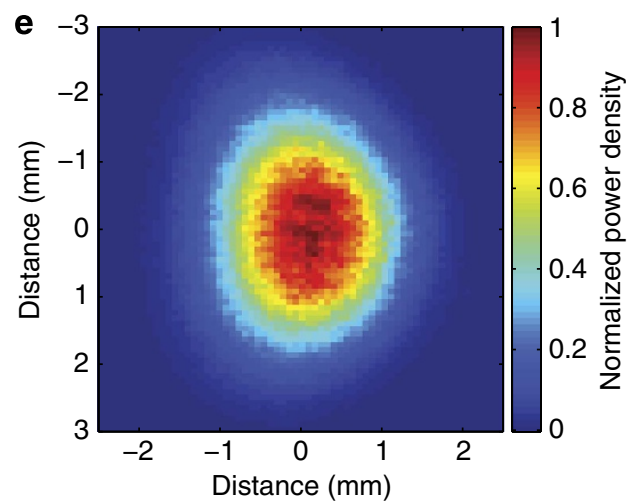

Figure 1 | Terahertz-driven linear accelerator. (a) Schematic of the THz LINAC. Top right: a linearly polarized THz pulse is converted into a radially polarized pulse by a segmented waveplate before being focused into the $\mathrm{THz}$ waveguide. The $\mathrm{THz}$ pulse is reflected at the end of the waveguide to co-propagate with the electron bunch, which enters the waveguide through a pinhole (lower left). The electron bunch is accelerated by the longitudinal electric field of the co-propagating $\mathrm{THz}$ pulse. The electron bunch exits the $\mathrm{THz}$ waveguide and passes through a hole in the focusing mirror (right) for the $\mathrm{THz}$ pulse. (b) Photograph of the compact millimetre scale THz LINAC. (c) The time-domain waveform of the THz pulse determined with electro-optic sampling (see Methods: Electro-optic sampling). Insert: corresponding frequency-domain spectrum. (d) The time-domain waveform of the THz pulse at the exit of a THz waveguide $5 \mathrm{~cm}$ in length, including two tapers. (e) Normalized intensity of the focused THz beam. 
precision corresponds to $10-\mathrm{fs}$ timing jitter, which is readily achievable ${ }^{22}$. On the other hand, the frequency is high enough that the plasma breakdown threshold for surface electric fields increases into the multi-GV m${ }^{-1}$ range $^{6}$. In addition, using optical generation techniques, we can have very short $\mathrm{THz}$ pulses $(\leq 100 \mathrm{ps})$ generated by picosecond lasers readily available at high average power and under rapid development. These short pulses allow for a limited amount of pulsed heating and a limited amount of average power loading at high repetition rates (on the order of $\mathrm{kHz}$ and above). Both the increase in operational frequency and reduction in pulse length will play a role in increasing the breakdown limit.

Here we report the experimental demonstration of electron acceleration using the axial component of an optically generated $10 \mu \mathrm{J} \mathrm{THz}$ pulse centred at $0.45 \mathrm{THz}$ (see Methods: $\mathrm{THz}$ pulse generation) in a waveguide. The $\mathrm{THz}$ pulse accelerates electrons in a circular waveguide consisting of a dielectric capillary with a metal outer boundary. The dielectric slows the group and phase velocity of the $\mathrm{THz}$ wave allowing it to accelerate low-energy electrons. We a observe a maximum energy gain of $7 \mathrm{keV}$ in $3 \mathrm{~mm}$. Future, $\mathrm{THz}$ accelerators designed for relativistic electron beams and using more intense $\mathrm{THz}$ sources will be able to reach $\mathrm{GeV} \mathrm{m}^{-1}$ accelerating gradients.

\section{Results}

Operation of the THz LINAC. A schematic view of the $\mathrm{THz}$ accelerator is shown in Fig. 1a with a photograph of the $\mathrm{THz}$
LINAC in Fig. 1b. Using 60-keV electrons, from a d.c. electron gun, an energy gain of $7 \mathrm{keV}$ is observed in a $3-\mathrm{mm}$ interaction length. The single-cycle $\mathrm{THz}$ pulse, see Fig. $1 \mathrm{c}-\mathrm{e}$, is produced via optical rectification of a $1.2 \mathrm{~mJ}, 1.03 \mu \mathrm{m}$ laser pulse with a $1 \mathrm{kHz}$ repetition rate (see Methods: Pump source). The $\mathrm{THz}$ pulse, whose polarization is converted from linear to radial by a segmented waveplate (see Methods: Radially polarized $\mathrm{THz}$ beam), is coupled into a waveguide with $10 \mathrm{MV} \mathrm{m}^{-1}$ peak on-axis electric field (see Methods: Structure testing). A 25-fC input electron bunch is produced with a $60-\mathrm{keV}$ d.c. photoemitting cathode excited by a 350 -fs ultraviolet pulse (see Methods: Ultraviolet photoemitter). The accelerating gradient in the $\mathrm{THz}$ structures demonstrated in this work can be as high as $\mathrm{GeV} \mathrm{m}^{-1}$ with a single-cycle $\mathrm{THz}$ pulse of $10 \mathrm{~mJ}$ (see Methods: $\mathrm{THz}$ LINAC), which can be readily produced by a $250-\mathrm{mJ}$ infrared pulse when using optimized $\mathrm{THz}$ generation ${ }^{23}$. Laser systems producing such and even higher energy picosecond pulses with up to $\mathrm{kHz}$ repetition rates are on the horizon ${ }^{24-26}$.

In this experiment, the $\mathrm{THz}$ waveguide supports a travelling $\mathrm{TM}_{01}$ mode that is phase matched to the velocity of the electron bunch produced by the d.c. photoinjector. It is the axial component of the $\mathrm{TM}_{01}$ mode that accelerates the electrons as they co-propagate down the waveguide. A travelling-wave mode is advantageous when considering the available single-cycle $\mathrm{THz}$ pulse because it does not require resonant excitation of the structure. A dielectric-loaded circular waveguide was selected due to the ease of fabrication in the $\mathrm{THz}$ band ${ }^{27}$. The inner diameter

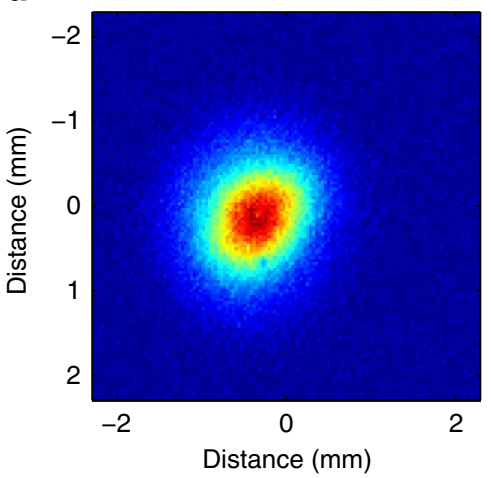

b

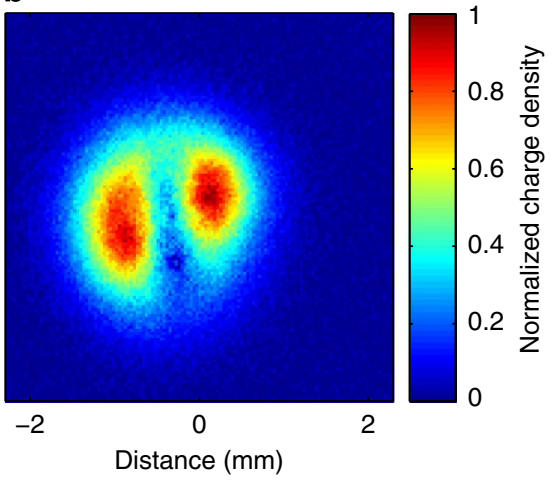

d

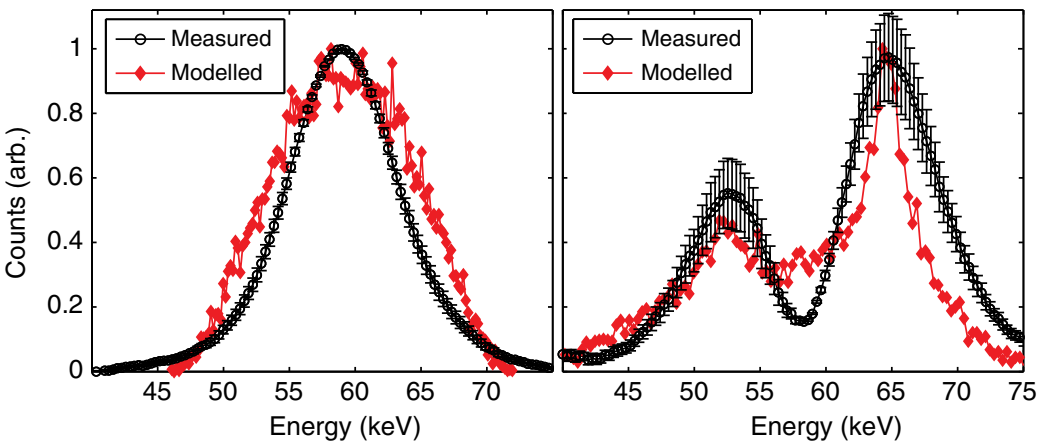

Figure 2 | Demonstration of terahertz acceleration. Transverse electron density of the electron bunch as recorded by a micro-channel plate (MCP) at $59 \mathrm{kV}$ for (a) THz off and (b) THz on. The bimodal distribution is due to the presence of accelerated and decelerated electrons, which are separated spatially by the magnetic dipole energy spectrometer. The images are recorded over a 3-s exposure at $1 \mathrm{kHz}$ repetition rate. (c) Comparison between simulated (red) and measured (black) energy spectrum of the electron bunch measured at the MCP due to the deflection of the beam by a magnetic dipole. At $59 \mathrm{keV}$ and with $25 \mathrm{fC}$ per bunch, the simulation predicts a $\sigma_{\perp}=513 \mu \mathrm{m}$ and $\Delta E=1.25 \mathrm{keV}$. The observed $\Delta E / E$ appears larger due to the large transverse size of the beam. After the pinhole, the transverse emittance is $25 \mathrm{~nm}$ rad and the longitudinal emittance is $5.5 \mathrm{~nm}$ rad. (d) Comparison between simulated (red) and measured (black) electron bunch at MCP after acceleration with THz. Decelerated electrons are present due to the long length of the electron bunch, as well as the slippage between the $\mathrm{THz}$ pulse and the electron bunch. Error bars in $\mathbf{c}$ and $\mathbf{d}$ correspond to one s.d. in counts over the data set of 10 integrated exposures. 

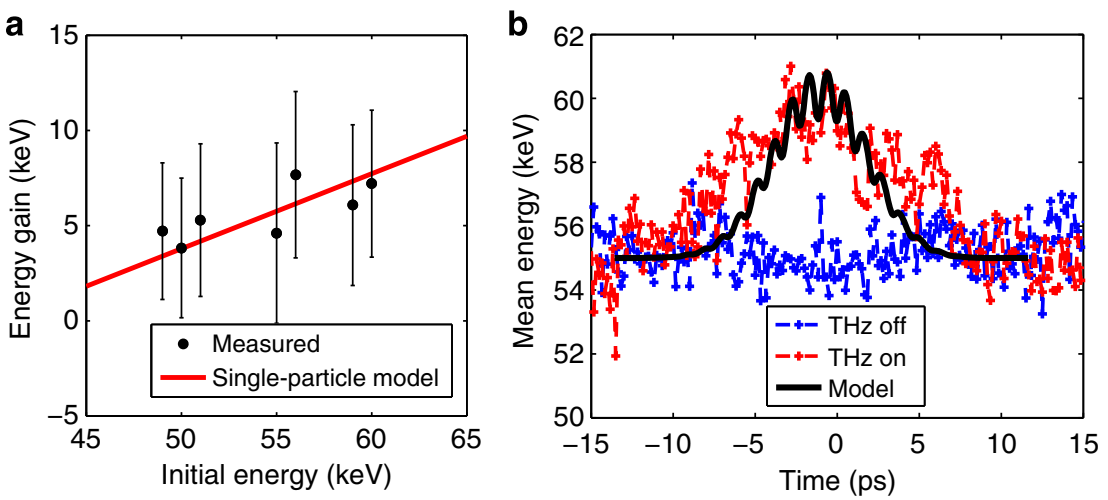

Figure 3 | Acceleration gradient and terahertz phasing. (a) Scaling of energy gain for accelerated electrons as a function of the initial electron energy at the entrance of the THz LINAC. Black dots with one s.d. error bars are measured values and the red line is a single-particle model. (b) The temporal profile for the mean energy gain of accelerated electrons comparing the $\mathrm{THz}$ on and $\mathrm{THz}$ off signal against the simulated electron bunch. The initial electron energy was set at $55 \mathrm{keV}$ to ensure stable performance of the d.c. electron gun over the acquisition time of the data set.

of the copper waveguide is $940 \mu \mathrm{m}$ with a dielectric wall thickness of $270 \mu \mathrm{m}$. This results in a vacuum space with a radius of $200 \mu \mathrm{m}$. The significant thickness of the dielectric is due to the low energy of the electrons entering the structure, and will decrease significantly at higher energy. One critical aspect for $\mathrm{THz}$ electron acceleration is proper interaction between the electron beam and the $\mathrm{THz}$ pulse. Coupling the radially polarized $\mathrm{THz}$ pulse into the single-mode dielectric waveguide was achieved with a centrally loaded dielectric horn. The design was optimized to maximize coupling with minimal fabrication complexity. Finite element electromagnetic simulations with HFSS ${ }^{28}$ indicate excellent coupling of the $\mathrm{THz}$ pulse over a $\sim 200 \mathrm{GHz}$ bandwidth (see Methods: Structure testing), which is compatible with the bandwidth of the radially polarized mode converter. The accelerating waveguide is $10 \mathrm{~mm}$ in length, including a single tapered horn for coupling the $\mathrm{THz}$ into the waveguide. Alignment between the $\mathrm{THz}$ waveguide and the d.c. gun is provided by a pin-hole aperture in a metal plate with a diameter of $100 \mu \mathrm{m}$ that abuts the waveguide. The $\mathrm{THz}$ pulse is coupled into the waveguide downstream of the accelerator and it propagates along the full length of the waveguide before being reflected by the pin-hole aperture, which acts as a short at $\mathrm{THz}$ frequencies. After being reflected the $\mathrm{THz}$ pulse co-propagates with the electron bunch. The low initial energy of the electrons results in the rapid onset of a phase-velocity mismatch between the electron bunch and the $\mathrm{THz}$ pulse once the electrons have been accelerated by the $\mathrm{THz}$ pulse and this limits the interaction length to $3 \mathrm{~mm}$ (see Methods: THz LINAC).

Observation of acceleration. The electron beam energy is determined via energy-dependent magnetic steering with a dipole located after the accelerator. Figure $2 \mathrm{a}, \mathrm{b}$ shows images of the electron beam produced by the micro-channel plate detector. The measured energy spectrum from the electron bunch with and without $\mathrm{THz}$ is shown in Fig. 2c,d for an initial mean energy of $59 \mathrm{keV}$. The curves are compared with PARMELA ${ }^{29}$ particle-incell (PIC) simulation results used to model the d.c. gun and the THz LINAC. The full width of the electron bunch length after the pinhole is $200 \mu \mathrm{m}$, which is long with respect to the wavelength of the $\mathrm{THz}$ pulse in the waveguide, $\lambda_{\mathrm{g}}=315 \mu \mathrm{m}$. The length of the electron bunch in combination with the phase-velocity mismatch between the electron bunch and the $\mathrm{THz}$ pulse results in the observation of both acceleration and deceleration of particles. With the available $\mathrm{THz}$ pulse energy, a peak energy gain of $7 \mathrm{keV}$ was observed by optimizing the electron beam voltage and timing of the $\mathrm{THz}$ pulse. The modelled curve in Fig. $2 \mathrm{~d}$ concurred with experiments for an on-axis electric field of $8.5 \mathrm{MV} \mathrm{m}^{-1}$. Using this estimated field strength, at the exit of the LINAC, the modelled transverse and longitudinal emittance are 240 and $370 \mathrm{~nm}$ rad, respectively. An increase in emittance from a transverse emittance of $25 \mathrm{~nm}$ rad and a longitudinal emittance of $5.5 \mathrm{~nm}$ rad after a pinhole located at the waveguide entrance is due to the long electron bunch length compared with the $\mathrm{THz}$ wavelength and can be easily remedied with a shorter ultraviolet pulse length.

Optimization of electron beam interaction with $\mathrm{THz}$ pulse. The energy gain achieved during the interaction of the electron bunch with the $\mathrm{THz}$ pulse is dependent on the initial energy of the electrons, because the set-up is operated in the non-relativistic limit where the velocity of the electrons varies rapidly. If the initial energy is decreased, the particle velocity decreases and the phase-velocity mismatch with the $\mathrm{THz}$ pulse increases reducing the interaction length and the acceleration of the particle. In Fig. 3a, the achieved mean output energy of the electron bunch is shown versus the initial energy. Higher initial energy was experimentally found to be favourable for higher energy gain. This observation is in agreement with a single-particle model ${ }^{30}$ for a peak on-axis electric field of $8.5 \mathrm{MV} \mathrm{m}^{-1}$ and an effective accelerating gradient of $2.5 \mathrm{MeVm}^{-1}$ (see Methods: $\mathrm{THz}$ LINAC). In Fig. 3b, the mean energy of accelerated electrons is shown as a function of the $\mathrm{THz}$ pulse delay for $55-\mathrm{keV}$ initial energy. The large temporal range of observed acceleration results from waveguide dispersion, which broadens the single-cycle pulse temporally as it propagates. Accelerated electrons are observed over the full range of the phase-matched $\mathrm{THz}$ pulse due to the long length of the electron bunch as it enters the $\mathrm{THz}$ waveguide. Modelling indicates that at the entrance of the $\mathrm{THz}$ waveguide for this initial energy, the electron bunch full width is $1.5 \mathrm{ps}$ in length, which is already a significant fraction of the $\mathrm{THz}$ cycle $(2.2 \mathrm{ps})$.

In conclusion, optically generated $\mathrm{THz}$ pulses were used to accelerate electrons in a simple and practical $\mathrm{THz}$ accelerator. An energy gain of $7 \mathrm{keV}$ was achieved over a 3-mm interaction length with good modelled emittance. Performance of these structures improves with an increase in electron energy and gradient making them attractive for compact accelerator applications. With upgrades to pump laser energy and technological improvements to $\mathrm{THz}$ sources ${ }^{31}$, laboratory demonstration of $\mathrm{GeV} \mathrm{m}^{-1}$ gradients in THz LINACs is realistic. Multi-GeV m ${ }^{-1}$ gradients and $>10 \mathrm{MeV}$ energy gain are achievable in dielectric-loaded circular waveguides ${ }^{30}$ with $10-\mathrm{mJ} \mathrm{THz}$ pulses and the injection of electrons at relativistic energies (see Methods: THz LINAC). 
a

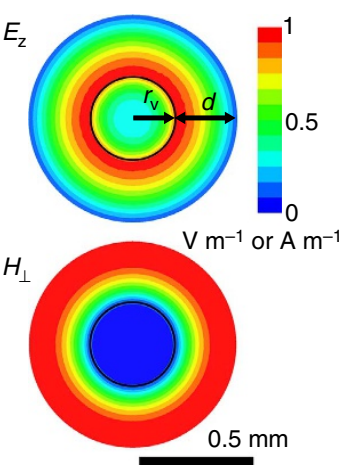

b

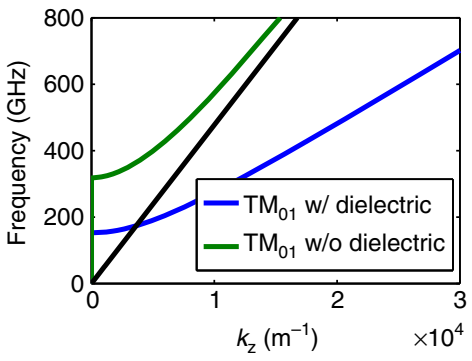

C

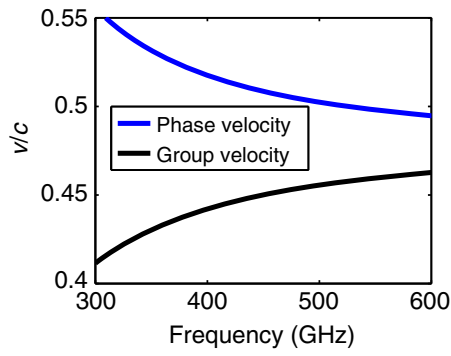

Figure 4 | TM $\mathbf{M}_{\mathbf{0 1}}$ THz LINAC parameters. (a) Normalized magnitude of the longitudinal electric field and perpendicular magnetic field for the TM $\mathrm{T}_{01}$ mode at $450 \mathrm{GHz}$ in a circular copper waveguide with dielectric loading. The inner diameter of the copper waveguide is $940 \mu \mathrm{m}$ with a vacuum radius $r_{\mathrm{v}}=200 \mu \mathrm{m}$ and a dielectric wall thickness of $d=270 \mu \mathrm{m}$. The dimensions $r_{\mathrm{v}}$ and $d$ are labelled in the $E_{\mathrm{z}}$ plot. The solid black line indicates the boundary between the vacuum core and the quartz capillary. (b) The dispersion relation for the TM01 mode with and without dielectric loading. The black line indicates the speed of light in vacuum. (c) The group and phase velocity of the $\mathrm{THz}$ pulse as a function of frequency with dielectric loading.

a

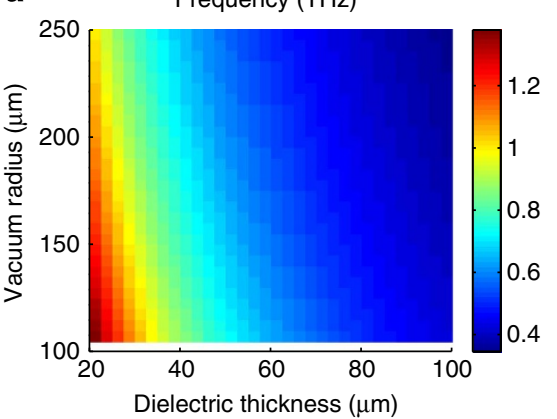

d

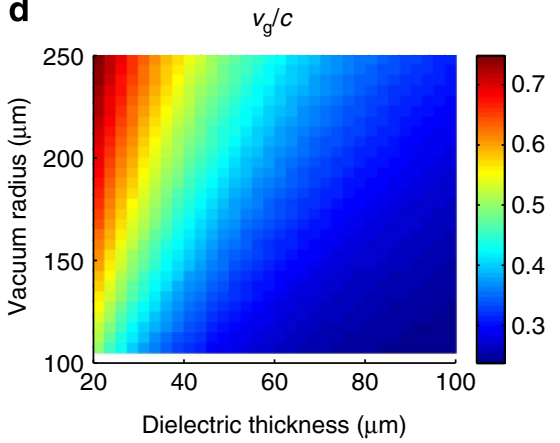

b

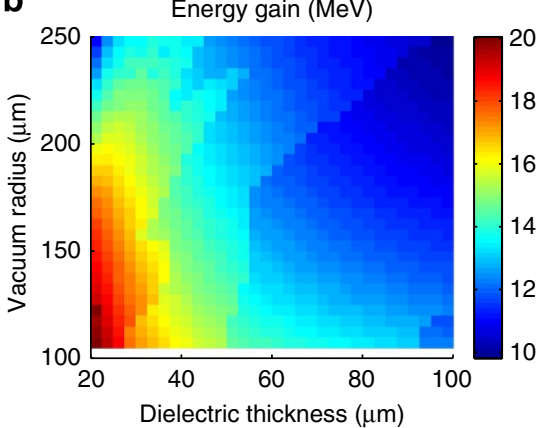

e

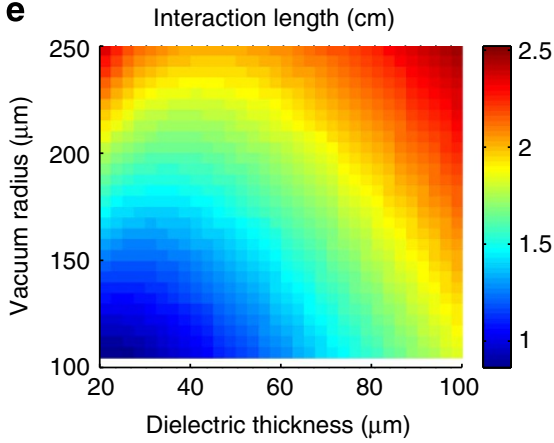

C

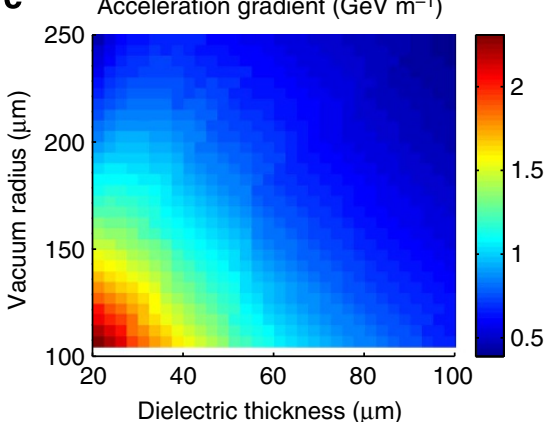

f

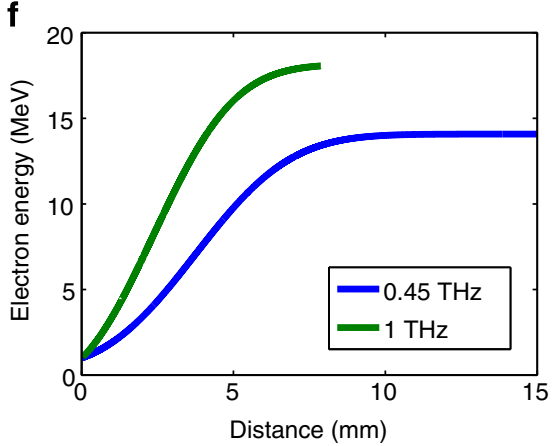

Figure 5 | Relativistic THz LINAC design. Performance parameters as a function of vacuum radius and dielectric wall thickness for a relativistic THz LINAC operating in the $\mathrm{TM}_{01}$ mode with a $10 \mathrm{~mJ}$ single-cycle $\mathrm{THz}$ pulse and an initial electron energy of $1 \mathrm{MeV}$. The phase velocity is $\mathrm{c}$ for the nominal frequency of operation. The (a) frequency of operation, (b) energy gain, (c) acceleration gradient, (d) group velocity and (e) interaction length for the THz LINAC. (f) The electron energy as a function of distance for two cases, which operate with a frequency of $0.45 / 1 \mathrm{THz}$, a vacuum space with a radius of $r_{\mathrm{v}}=105 / 105 \mu \mathrm{m}$ and a dielectric wall thickness of $90 / 30 \mu \mathrm{m}$.

The available THz pulse energy scales with infrared pump energy, with recently reported results of $\mathrm{mJ} \mathrm{THz}$ pulse energies and $\sim 3 \%$ infrared-to-THz conversion efficiencies ${ }^{32,33}$. Multiple stages of $\mathrm{THz}$ acceleration can be used to achieve higher energy gain with additional infrared pump lasers for subsequent stages. Timing jitter will improve on the jitter of conventional accelerators since the accelerating field and photoemitting pulse are produced by the same drive laser. Therefore, one expects the resulting electron bunch to have tighter synchronization than possible in today's RF-based accelerators, where the photoemitting laser pulse is synchronized to the RF drive by standard RF techniques (that is, phase locked loops operating at $\mathrm{GHz}$ speeds). This proof-ofprinciple $\mathrm{THz}$ linear accelerator demonstrates the potential for an all-optical acceleration scheme that can be readily integrated into small-scale laboratories providing users with electron beams that will enable new experiments in ultrafast electron diffraction and $\mathrm{X}$-ray production.

\section{Methods}

THz pulse generation. The $\mathrm{THz}$ pulse that is used in the accelerating structure is generated with optical rectification of $700 \mathrm{fs}, 1.03 \mu \mathrm{m}$ pulses in cryogenically cooled stoichiometric lithium niobate (LN) doped with $1 \% \mathrm{MgO}$. LN was chosen because it exhibits multiple advantages with low $\mathrm{THz}$ absorption, large bandgap, a high damage threshold and a high effective nonlinear coefficient, that is, large $d_{\text {eff. }}$.

For a high efficiency of conversion in LN, a tilted-pulse-front pumping scheme ${ }^{23}$ is required. The $\mathrm{THz}$ pulse is centred at $0.45 \mathrm{THz}$ with a broad spectrum ranging from 0.2 to $0.8 \mathrm{THz}$. A THz pulse energy of $10 \mu \mathrm{J}$ is produced from $1.2 \mathrm{~mJ}$ of NIR, which is slightly lower than the peak conversion efficiency of $\mathrm{THz}$ generation ${ }^{23}$ due to a larger spot size in the LN (decreased fluence) for improved transport of the 

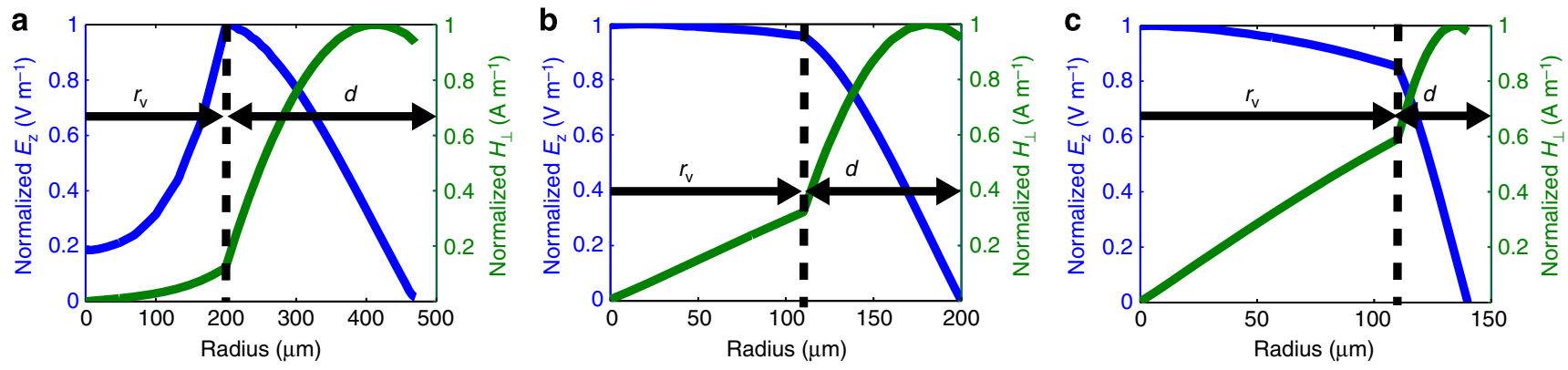

Figure 6 | Electromagnetic field distribution. The field distribution in the THz waveguide for (a) the non-relativistic design described in Fig. 4 with $r_{\mathrm{v}}=200 \mu \mathrm{m}$ and $d=270 \mu \mathrm{m}$, (b) the relativistic design in Fig. $5 \mathrm{f}$ for operation at $0.45 \mathrm{THz}$ with $r_{\mathrm{v}}=105 \mu \mathrm{m}$ and $d=90 \mu \mathrm{m}$ and (c) the relativistic design in Fig. $5 \mathrm{f}$ for operation at $1 \mathrm{THz}$ with $r_{\mathrm{v}}=105 \mu \mathrm{m}$ and $d=30 \mu \mathrm{m}$.
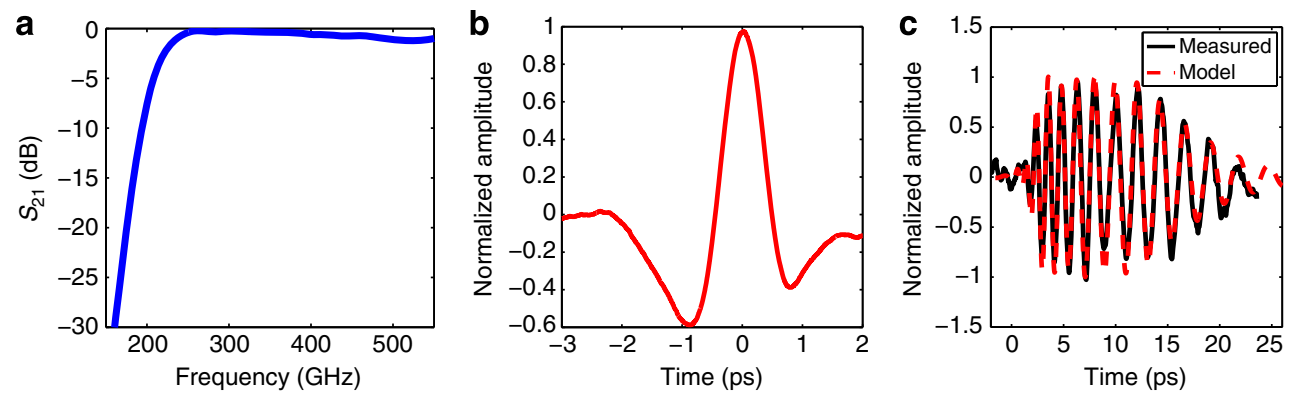

Figure 7 | Coupling and dispersion of THz pulses in waveguides. (a) HFSS ${ }^{28}$ simulation of the coupling of the free-space radially polarized mode into the $\mathrm{TM}_{01}$ mode through a dielectric-loaded taper. (b) The time-domain waveform of the $\mathrm{THz}$ pulse determined with electro-optic sampling before being coupled into the $\mathrm{THz}$ waveguide. (c) The measured versus the modelled time-domain waveform of the $\mathrm{THz}$ pulse at the exit of a 5-cm (including tapers) dielectric-loaded $\mathrm{THz}$ waveguide.

$\mathrm{THz}$ beam. The $\mathrm{THz}$ beam has excellent Gaussian mode content, which allows for low-loss coupling. The THz beam was characterized spatially by a pyroelectric detector array (Spiricon Pyrocam IV, Ophir Photonics).

Pump source. The pump source for THz generation is a $\mathrm{Yb}: \mathrm{KYW}$ chirped pulse regenerative amplifier (RGA) producing $1.5-\mathrm{mJ}$ pulses with $1 \mathrm{kHz}$ repetition rate at a centre wavelength of $1,030 \mathrm{~nm}$ and bandwidth of $2.1 \mathrm{~nm}$. The dielectric grating compressor following the RGA compresses the pulses to a near-transform-limited 700 -fs pulse duration (full width at half maximum). The seed for the RGA was a mode-locked Yb-doped fibre oscillator emitting $70 \mathrm{fs}, 0.2 \mathrm{~nJ}$ pulses at $80 \mathrm{MHz}$ (ref. 34) amplified to $1.6 \mathrm{~nJ}$ by a Yb-doped fibre amplifier. After losses through the optical elements in the pulse front tilting set-up including a diffraction grating, the impinging pump energy into the (LN) crystal was $1.2 \mathrm{~mJ}$.

Ultraviolet photoemitter. About $2 \%$ of the available 1,030 nm NIR pump energy was used to generate the ultraviolet photoemitter pulses by fourth harmonic generation from $1.03 \mu \mathrm{m}$ based on two-stage second harmonic generation in a 10-mm-long type I LBO crystal for infrared to green and in a 0.5 -mm-long type-I $\mathrm{BBO}$ crystal for the green to ultraviolet, respectively. A BG-39 band-pass filter was used to remove the fundamental NIR component after the first stage. The ultraviolet pulses have a duration of $\sim 350 \mathrm{fs}$ and are focused onto the photocathode with a beam waist of $\sim 200 \mu \mathrm{m}$. Since both the $\mathrm{THz}$ and ultraviolet pulses are produced from the same sub-picosecond NIR laser, the timing jitter between them is negligible.

Electro-optic sampling. Electro-optic sampling was used to determine the temporal and spectral properties of the THz pulse and dispersion induced from the quasi-optical elements in the $\mathrm{THz}$ beamline. Optical synchronization between the $\mathrm{THz}$ pulse and the mode-locked Yb-doped fibre seed oscillator $(80 \mathrm{MHz}, 70 \mathrm{fs}$, $1,030 \mathrm{~nm}$ ) was ensured as explained in the Pump source section of Methods. Birefringence was induced in a $200-\mu \mathrm{m}$ thick, 110 -cut ZnTe crystal. A quarter-wave plate followed by a polarizer converts the field-induced birefringence to an intensity modulation, and the intensity modulation is measured using a balanced detector with a delay scan.

Radially polarized THz beam. The THz pulse generated by optical rectification is linearly polarized, which is not compatible with the $\mathrm{TM}_{01}$ mode used in the accelerating structure. A segmented half waveplate was used to convert the linearly polarized light to radially polarized light ${ }^{35}$. Each segment of the waveplate imparts the appropriate rotation to the polarization to transition from a linear to a radially polarized beam, which couples well in the far field to the $\mathrm{TM}_{01}$ mode of the accelerating structure. A segmented $\lambda / 2$ waveplate with eight segments of $\sim 8$-mm-thick quartz designed for operation at $0.45 \mathrm{THz}$ was used.

THz LINAC. The THz pulse accelerates electrons in a circular waveguide consisting of a quartz capillary inserted into a hollow copper cylinder ${ }^{36}$, see Fig. 1a. The inner diameter of the copper waveguide is $940 \mu \mathrm{m}$ with a dielectric wall thickness of $d=270 \mu \mathrm{m}$. This results in a vacuum space with a radius of $r_{\mathrm{v}}=200 \mu \mathrm{m}$. The dielectric constant of the quartz capillary is nominally $\varepsilon=4.41$. The operational mode of the LINAC is a travelling $\mathrm{TM}_{01}$ mode, see Fig. $4 \mathrm{a}$. The dispersion relation for the operating mode is shown in Fig. 4b. At the centre frequency of the $\mathrm{THz}$ pulse $(450 \mathrm{GHz})$, the group velocity is $v_{\mathrm{g}} / c=0.46$ and the phase velocity is $v_{\mathrm{p}} / c=0.505$.

Due to the operational frequency's proximity to the cutoff of the waveguide, the accelerating mode is highly dispersive with the phase and group velocity shown in Fig. $4 \mathrm{c}$ as a function of frequency. The waveguide dimensions of the LINAC were chosen to optimize for this experimental set-up with a low initial electron energy of $60 \mathrm{keV}$, the $\mathrm{THz}$ pulse energy available and the transverse dimension of the electron beam. At the nominal $60 \mathrm{keV}$, the electron velocity is $v / c=0.45$. This velocity is increased as the particles are accelerated increasing the electron velocity, however, the mismatch with the phase velocity results in an interaction length of $3 \mathrm{~mm}$. At this point, the electron bunch and $\mathrm{THz}$ pulse interaction is terminated by the presence of the taper, which rapidly reduces the intensity of the on-axis electric field as the waveguide diameter is increase. This slippage causes a peak on-axis electric field of $8.5 \mathrm{MV} \mathrm{m}^{-1}$ to produce an accelerating gradient of $2.5 \mathrm{MeV} \mathrm{m}^{-1}$ at the nominal initial energy of $60 \mathrm{keV}$.

With increased THz energy and increased electron energy, one can consider a relativistic accelerator design in which the phase velocity of the $\mathrm{TM}_{01}$ mode is equal to the speed of light. In addition, the design of the waveguide can be optimized to match the frequency of the available source. Figure 5a-e presents the frequency of operation, energy gain, acceleration gradient, group velocity and interaction length as a function of vacuum radius and dielectric thickness assuming a $10-\mathrm{mJ}$ singlecycle $\mathrm{THz}$ pulse and an initial electron energy of $1 \mathrm{MeV}$. Figure $5 \mathrm{f}$ presents the electron energy as a function of distance for two cases, which operate with a frequency of $0.45 / 1 \mathrm{THz}$, a vacuum space with a radius of $r_{\mathrm{v}}=105 / 105 \mu \mathrm{m}$ and a dielectric wall thickness of $d=90 / 30 \mu \mathrm{m}$. The drastic increase in the acceleration gradients shown in Fig. 5 is not only due to the increased THz pulse energy. The performance of a travelling-wave $\mathrm{THz}$ accelerator structure greatly increases when the electron velocity approaches the speed of light, because there is less dispersion for the $\mathrm{THz}$ pulse, a longer interaction length, the waveguide radius is decreased, the amount of dielectric material is greatly reduced and the electric field profile 
is improved. Figure 6 compares the field distribution for the non-relativistic waveguide design (Fig. 4), which was investigated experimentally and the relativistic designs highlighted in Fig. 5 f. Note the decrease in waveguide radius, the decrease in dielectric wall thickness and the improved relative amplitude of the longitudinal electric field in the accelerating region of the waveguide $(r<100 \mu \mathrm{m})$ for the two relativistic $\left(v_{\mathrm{p}}=c\right)$ designs, which all contribute to improve the efficiency of the accelerator. The transverse fields in all cases are localized to the regions without the presence of the electron bunch alleviating concerns with the potential for ponderomotive effects. The use of multi-mJ THz pulses will increase the peak electric fields in these waveguides well above a $\mathrm{GV} \mathrm{m}^{-1}$; however, the long wavelength and short propagation distance prevent the onset of nonlinearities such as self phase modulation ${ }^{37,38}$. Further details and numerical studies for relativistic THz LINACs using dielectric-loaded waveguides and few-cycle $\mathrm{THz}$ pulses were previously reported in ref. 30 .

Structure testing. A THz waveguide structure consisting of two tapers separated by a uniform waveguide section was built to test and optimize waveguide design by performing transmitted energy and polarization measurements. Measurements were performed with a Gentec-EO Pyroelectric Joulemeter Probe that is capable of measuring pulse energies exceeding $100 \mathrm{~nJ}$. Efficient excitation of the $\mathrm{TM}_{01}$ mode, demonstrated in these measurements, is a critical requirement in developing a compact high-gradient THz LINAC. As a first step, the linearly polarized beam is converted to a radially polarized beam with a segmented waveplate. The vertical and horizontal polarization measured after the segmented waveplate were $53 \%$ and $47 \%$, respectively. This radially polarized beam was coupled into a test waveguide structure that was $5 \mathrm{~cm}$ in length, including two tapers to couple the $\mathrm{THz}$ pulse into and out of the waveguide. The long length was selected to demonstrate that ohmic losses are manageable even over significant interaction lengths. The simulated coupling for the THz pulse into the waveguide is shown in Fig. 7a with a bandwidth of over $200 \mathrm{GHz}$. The power coupled through the structure was measured with and without dielectric loading at $32 \%$ and $54 \%$, respectively. The increased losses in the dielectric-loaded waveguide are due to increased ohmic losses. For the dielectric-loaded waveguide, $2 \mu \mathrm{J}$ of energy was measured at the exit of the waveguide, which corresponds to a calculated peak on-axis electric field is $9.7 \mathrm{MV} \mathrm{m}^{-1}$.

Electro-optic sampling (see Methods: Electro-optic sampling) was used to characterize the linear dispersion of the dielectric-loaded waveguide test structure, which has the same dimensions as the waveguide used in the acceleration experiment and the properties shown in Fig. $4 \mathrm{~b}, \mathrm{c}$. Figure $7 \mathrm{~b}$ shows the timedomain spectrum produced by the $\mathrm{THz}$ source (see Methods: $\mathrm{THz}$ pulse generation). Figure $7 \mathrm{c}$ shows a comparison between the measured time-domain spectrum of THz pulse at the exit of the waveguide with the simulated propagation of the input $\mathrm{THz}$ pulse propagated with the dispersive parameters of the waveguide design provided in Fig. 4b,c. Excellent agreement indicates that the fabricated structure has the desired performance.

\section{References}

1. Emma, P. et al. First lasing and operation of an ångstrom-wavelength free-electron laser. Nat. Photon. 4, 641-647 (2010).

2. Ishikawa, T. et al. A compact X-ray free-electron laser emitting in the sub-angstrom region. Nat. Photon. 6, 540-544 (2012).

3. Kilpatrick, W. Criterion for vacuum sparking designed to include both $\mathrm{rf}$ and dc. Rev. Sci. Instrum. 28, 824-826 (1957).

4. Wang, J. \& Loew, G. in Proceedings of the 1989 IEEE (Particle Accelerator Conference, 1989. Accelerator Science and Technology) 1137-1139 (Stanford, CA, USA, 1989).

5. Forbes, R. G. \& Deane, J. H. Reformulation of the standard theory of fowlernordheim tunnelling and cold field electron emission. Proc. R. Soc. Lond. A 463, 2907-2927 (2007).

6. Thompson, M. et al. Breakdown limits on gigavolt-per-meter electron-beamdriven wakefields in dielectric structures. Phys. Rev. Lett. 100, 214801 (2008).

7. Geddes, C. et al. High-quality electron beams from a laser wakefield accelerator using plasma-channel guiding. Nature 431, 538-541 (2004).

8. Plettner, T. et al. Visible-laser acceleration of relativistic electrons in a semi-infinite vacuum. Phys. Rev. Lett. 95, 134801 (2005).

9. Hafz, N. A. et al. Stable generation of GeV-class electron beams from self-guided laser-plasma channels. Nat. Photon. 2, 571-577 (2008).

10. Plettner, T. \& Byer, R. Proposed dielectric-based microstructure laser-driven undulator. Phys. Rev. Spec. Top. Accel. Beams 11, 030704 (2008).

11. Kneip, S. et al. Near-GeV acceleration of electrons by a nonlinear plasma wave driven by a self-guided laser pulse. Phys. Rev. Lett. 103, 035002 (2009).

12. Varin, C. \& Piché, M. Acceleration of ultra-relativistic electrons using high-intensity tm01 laser beams. Appl. Phys. B 74, s83-s88 (2002).

13. Payeur, S. et al. Generation of a beam of fast electrons by tightly focusing a radially polarized ultrashort laser pulse. Appl. Phys. Lett. 101, 041105 (2012).

14. Peralta, E. et al. Demonstration of electron acceleration in a laser-driven dielectric microstructure. Nature 503, 91-94 (2013).

15. Breuer, J. \& Hommelhoff, P. Laser-based acceleration of nonrelativistic electrons at a dielectric structure. Phys. Rev. Lett. 111, 134803 (2013).
16. Carbajo, S., Wong, L. J., Nanni, E., Schimpf, D. N. \& Kärtner, F. X. in High Intensity Lasers and High Field Phenomena, HTu2C-6 (Optical Society of America, 2014).

17. Faure, J. et al. A laser-plasma accelerator producing monoenergetic electron beams. Nature 431, 541-544 (2004).

18. Yoder, R. \& Rosenzweig, J. Side-coupled slab-symmetric structure for highgradient acceleration using terahertz power. Phys. Rev. Spec. Top. Accel. Beams 8, 111301 (2005).

19. Hebling, J. et al.Optical manipulation of relativistic electron beams using Thz pulses. Preprint at http://arxiv.org/abs/1109.6852 (2011).

20. Pálfalvi, L., Fülöp, J., Tóth, G. \& Hebling, J. Evanescent-wave proton postaccelerator driven by intense thz pulse. Phys. Rev. Spec. Top. Accel. Beams 17, 031301 (2014).

21. Wimmer, L. et al. Terahertz control of nanotip photoemission. Nat. Phys. 10, 432-436 (2014).

22. Schibli, T. et al. Attosecond active synchronization of passively mode-locked lasers by balanced cross correlation. Opt. Lett. 28, 947-949 (2003).

23. Huang, S.-W. et al. High conversion efficiency, high energy terahertz pulses by optical rectification in cryogenically cooled lithium niobate. Opt. Lett. 38, 796-798 (2013).

24. Reagan, B. A. et al. Demonstration of a $100 \mathrm{hz}$ repetition rate gain-saturated diode-pumped table-top soft X-ray laser. Opt. Lett. 37, 3624-3626 (2012).

25. Zapata, L. E. et al. Cryogenic yb: Yag composite-thin-disk for high energy and average power amplifiers. Opt. Lett. 40, 2610-2613 (2015).

26. Klingebiel, S. et al. in CLEO: Science and Innovations, STu4O-2 (Optical Society of America, 2015).

27. Mitrofanov, O., James, R., Fernández, F. A., Mavrogordatos, T. K. \& Harrington, J. A. Reducing transmission losses in hollow thz waveguides. IEEE Trans. THz Sci. Technol. 1, 124-132 (2011).

28. ANSYS HFSS. Academic Research, Release v15.0 (Ansys Inc., 2013).

29. Young, L. \& Billen, J. in Proceedings of the 2003 IEEE Particle Accelerator Conference (PAC 03) (The particle tracking code parmela) (Portland, Oregon, 2003).

30. Wong, L. J., Fallahi, A. \& Kärtner, F. X. Compact electron acceleration and bunch compression in THz waveguides. Opt. Express 21, 9792-9806 (2013).

31. Chen, Z., Zhou, X., Werley, C. A. \& Nelson, K. A. Generation of high power tunable multicycle teraherz pulses. Appl. Phys. Lett. 99, 071102 (2011).

32. Vicario, C. et al. Generation of $0.9-\mathrm{mj}$ thz pulses in dstms pumped by a Cr: $\mathrm{Mg}_{2} \mathrm{SiO}_{4}$ laser. Opt. Lett. 39, 6632-6635 (2014).

33. Fülöp, J. A. et al. in CLEO: Science and Innovations, SW1F-5 (Optical Society of America, 2014).

34. Hong, K.-H. et al. Generation of $287 \mathrm{~W}, 5.5 \mathrm{ps}$ pulses at $78 \mathrm{MHz}$ repetition rate from a cryogenically cooled Yb:YAG amplifier seeded by a fiber chirped-pulse amplification system. Opt. Lett. 33, 2473-2475 (2008).

35. Grosjean, T. et al. Linear to radial polarization conversion in the thz domain using a passive system. Opt. Express 16, 18895-18909 (2008).

36. Miyagi, M. \& Kawakami, S. Design theory of dielectric-coated circular metallic waveguides for infrared transmission. J. Lightwave Technol. 2, 116-126 (1984)

37. Zalkovskij, M. et al. Terahertz-induced kerr effect in amorphous chalcogenide glasses. Appl. Phys. Lett. 103, 221102 (2013).

38. Harbold, J. et al. Highly nonlinear as-s-se glasses for all-optical switching. Opt. Lett. 27, 119-121 (2002).

\section{Acknowledgements}

We acknowledge W.S. Graves for many discussions and help with Parmela simulations; L.J. Wong for discussions on THz waveguides used for acceleration; S.-W. Huang for his assistance with $\mathrm{THz}$ generation; and $\mathrm{L}$. Hua for his assistance with the operation of the laser system. This work was supported by DARPA under contract N66001-11-1-4192, by AFOSR under grant AFOSR-A9550-12-1-0499, DOE DE-FG02-10ER46745, DOE DE-FG02-08ER41532, ERC Synergy Grant 609920 and NSF DMR-1042342, the Center for Free-Electron Laser Science at DESY and the excellence cluster The Hamburg Centre for Ultrafast Imaging-Structure, Dynamics and Control of Matter at the Atomic Scale of the Deutsche Forschungsgemeinschaft.

\section{Author contributions}

E.A.N., A.F. and F.X.K. conceived and designed the experiment. E.A.N. built and performed the experiment with the help from W.R.H. and K.-H.H., A.F. and E.A.N. provided the design for the dielectric-loaded metal waveguide. W.R.H. and K.R. provided the terahertz source. G.M. and R.J.D.M. were responsible for the d.c. gun. W.R.H., K.R., K.-H.H., A.F. and F.X.K. provided feedback to improve the experiment. E.A.N. developed and performed the simulations for data evaluation and interpretation of results. E.A.N. wrote the manuscript with contributions from W.R.H., K.R. and K.-H.H and revisions by all. K.-H.H. and F.X.K. provided management and oversight to the project.

\section{Additional information}

Competing financial interests: The authors declare no competing financial interests. 
Reprints and permission information is available online at http://npg.nature.com/ reprintsandpermissions/

How to cite this article: Nanni, E. A. et al. Terahertz-driven linear electron acceleration. Nat. Commun. 6:8486 doi: 10.1038/ncomms9486 (2015) (c) (i) This work is licensed under a Creative Commons Attribution 4.0 International License. The images or other third party material in this article are included in the article's Creative Commons license, unless indicated otherwise in the credit line; if the material is not included under the Creative Commons license users will need to obtain permission from the license holder to reproduce the material. To view a copy of this license, visit http://creativecommons.org/licenses/by/4.0/ 